\title{
Site-directed mutagenesis of the quorum-sensing transcriptional regulator SinR affects the biosynthesis of menaquinone in Bacillus subtilis
}

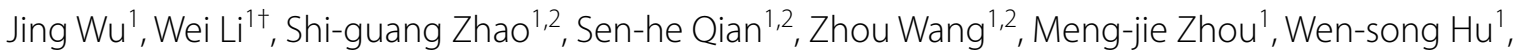
Jian Wang ${ }^{1}$, Liu-xiu Hu ${ }^{1,3}$, Yan Liu ${ }^{1,2^{*}}$ (D) and Zheng-lian Xue , $^{1,2^{*}}$

\begin{abstract}
Background: Menaquinone (MK-7) is a highly valuable vitamin $\mathrm{K}_{2}$ produced by Bacillus subtilis. Common static metabolic engineering approaches for promoting the production of MK-7 have been studied previously. However, these approaches caused an accumulation of toxic substances and reduced product yield. Hence, dynamic regulation by the quorum sensing (QS) system is a promising method for achieving a balance between product synthesis and cell growth.

Results: In this study, the QS transcriptional regulator SinR, which plays a significant role in biofilm formation and MK production simultaneously, was selected, and its site-directed mutants were constructed. Among these mutants, $\sin R$ knock out strain (KO-SinR) increased the biofilm biomass by 2.8-fold compared to the wild-type. SinR ${ }^{\text {quad }}$ maximized the yield of MK-7 (102.56 $\pm 2.84 \mathrm{mg} / \mathrm{L})$. To decipher the mechanism of how this mutant regulates MK-7 synthesis and to find additional potential regulators that enhance MK-7 synthesis, RNA-seq was used to analyze expression changes in the QS system, biofilm formation, and MK-7 synthesis pathway. The results showed that the expressions of tapA, tas $A$ and epsE were up-regulated 9.79-, 0.95-, and 4.42-fold, respectively. Therefore, SinR ${ }^{\text {quad }}$ formed more wrinkly and smoother biofilms than BS168. The upregulated expressions of $g / p F, g / p k$, and $g / p D$ in this biofilm morphology facilitated the flow of glycerol through the biofilm. In addition, NADH dehydrogenases especially $s d h A$, sdhB, sdhC and $g / p D$, increased 1.01-, 3.93-, 1.87-, and 1.11-fold, respectively. The increased expression levels of NADH dehydrogenases indicated that more electrons were produced for the electron transport system. Electrical hyperpolarization stimulated the synthesis of the electron transport chain components, such as cytochrome $c$ and MK, to ensure the efficiency of electron transfer. Wrinkly and smooth biofilms formed a network of interconnected channels with a low resistance to liquid flow, which was beneficial for the uptake of glycerol, and facilitated the metabolic flux of four modules of the MK-7 synthesis pathway.
\end{abstract}

Conclusions: In this study, we report for the first time that SinR ${ }^{\text {quad }}$ has significant effects on MK-7 synthesis by forming wrinkly and smooth biofilms, upregulating the expression level of most NADH dehydrogenases, and providing higher membrane potential to stimulate the accumulation of the components in the electron transport system.

\footnotetext{
*Correspondence: liuyan@ahpu.edu.cn; xuezl@ahpu.edu.cn

'Wei Li: the same contribution to the paper

${ }^{1}$ College of Biology and Food Engineering, Anhui Polytechnic University,

Wuhu 241000, China

Full list of author information is available at the end of the article
}

(C) The Author(s) 2021. This article is licensed under a Creative Commons Attribution 4.0 International License, which permits use, sharing, adaptation, distribution and reproduction in any medium or format, as long as you give appropriate credit to the original author(s) and the source, provide a link to the Creative Commons licence, and indicate if changes were made. The images or other third party material in this article are included in the article's Creative Commons licence, unless indicated otherwise in a credit line to the material. If material is not included in the article's Creative Commons licence and your intended use is not permitted by statutory regulation or exceeds the permitted use, you will need to obtain permission directly from the copyright holder. To view a copy of this licence, visit http://creativeco mmons.org/licenses/by/4.0/. The Creative Commons Public Domain Dedication waiver (http://creativecommons.org/publicdomain/ zero/1.0/) applies to the data made available in this article, unless otherwise stated in a credit line to the data. 
Keywords: Bacillus subtilis, Menaquinone, Transcriptional regulator, Site-directed mutagenesis, SinR

\section{Introduction}

As a highly valuable vitamin $\mathrm{K}_{2}$, menaquinone-7 (MK7) is a polyene compound consisting of a 2-methyl1,4-naphthoquinone ring structure with a side chain of seven isoprene units [1-3]. It was reported that MK-7 is a component of microbial plasma membranes and plays an important role in electron transport and oxidative phosphorylation $[4,5]$. Owing to its good bioavailability, MK-7 functions in protecting human health [6] by preventing osteoporosis [7], arterial calcification, cardiovascular disease, and Parkinson disease $[8,9]$. The cost of treating osteoporosis and cardiovascular diseases is high and will likely increase with the aging of populations. The global osteoporosis drug market in 2009 was estimated to be $\$ 8-9.4$ billion, and the market for cardiovascular drugs was estimated to be $\$ 110-140$ billion [1]. Thus, there is a large market for $\mathrm{MK}$ and the biosynthesis of MK-7 has received much attention from academia and industry.

In the past decades, many studies have focused on improving the production of MK-7 by microbial fermentation [10-12]. For example, medium components, culture conditions, additives, screening technologies, and the overexpression of exogenous genes in metabolic pathways [13-15] were studied to promote the production of MK. However, these common static metabolic engineering approaches tend to interrupt the elementary metabolic network, resulting in reduced substrate conversion, the accumulation of toxic substances, or reduced product yield [16], the maximum yield of MK-7 was only $35.5 \mathrm{mg} / \mathrm{L}$ [17]. Hence, compared with static regulation, dynamic regulation is a promising method achieving a balance between product synthesis and cell growth [18]. Engineered dynamic regulation systems with their capability of adapting to complicated extracellular or intracellular environments are valuable for fine-tuning metabolic flux $[19,20]$.

In previous studies, dynamic regulation systems have been classified into three categories: biosensors, metabolites response promoters, and quorum-sensing (QS) systems [21]. Biosensors derived from transcription factors bind to DNA to regulate gene expression when interacting with metabolites [22-24]. For example, Xu et al. constructed the malonyl-CoA-responsive biosensors, which could dynamically compensate for the key enzymes in Escherichia coli (E. coli) to achieve a metabolic balance between cell growth and product formation. They ultimately improved the fatty acid titer 2.1-fold [22]. Metabolite responsive promoters are commonly recognized by screening local promoters that respond to a particular metabolite and regulate gene expression. For instance, Dahl et al. regulated farnesyl pyrophosphate production in the isoprenoid biosynthetic pathway by applying the farnesyl pyrophosphate-responsive promoter. The production of amorphadiene in E. coli was improved twofold [25]. The potency of the target product can be greatly increased by using biosensors and metabolic reaction promoters. However, these promoters exist in pathwayspecific regulatory systems, severely limiting their widespread applications in many other metabolic pathways [26]. The quorum sensing (QS) system can regulate gene expression according to changes in cell density and overcome the disadvantages of transcriptional factor-based biosensors and metabolite-responsive promoters [27]. The QS system is not dependent on inducers, interventions, or metabolic pathways but rewires the control processes to depend on cell density [28]. For instance, to avoid the toxicity of heterogeneous pathways on cells, an Esa QS circuit with activation and inhibition functions was put forward to produce metabolites without inducers. With the activation of the QS system and dynamic regulation of the biosynthetic pathway by Esa-PesaR, the titer of 4-hydroxyphenylacetic acid increased by $46.4 \%$ compared with the static control pathway in E. coli [29]. This work proved that QS systems are helpful for finetuning metabolic networks.

B. subtilis has two QS systems that employ two different processed peptide autoinducers-ComX and competence stimulating peptide (CSP) - to regulate competence and sporulation processes [30]. To explore whether the QS system could promote MK-7 production and how the QS system regulates MK-7 synthesis, a regulator in the QS system which that plays significant role in biofilm formation and MK production simultaneously was selected and its site-directed mutants were constructed. To decipher the mechanism of how this mutant regulates MK-7 synthesis and find additional potential regulators that enhance MK-7 synthesis, the transcriptome was analyzed for expression changes in the QS system, biofilm formation, and MK-7 synthesis pathway of the different mutants. All these results demonstrate that a more wrinkly and smoother biofilm facilitated the flow of glycerol through the biofilm and enhanced MK-7 synthesis. In addition, the electrical hyperpolarization stimulated the synthesis of the electron transport chain components, such as cytochrome $\mathrm{c}$ and MK, to ensure the efficiency of electron transfer. This study also proposed some potential regulators of MK-7 biosynthesis, which could provide 
more ideas for the further enhancement of MK-7 production and deepen our understanding of the molecular mechanism of MK biosynthesis.

\section{Materials and methods}

\section{Gene knockout, site-directed mutagenesis and growth conditions}

The method used for gene knockout and overexpression was similar to that used by [31]. Briefly, the upstream and downstream sequences (both $\sim 1000 \mathrm{bp}$ ) flanking the deletion targets were amplified from $B$. subtilis. These two fragments and the lox71-zeo-lox66 cassettes, which were amplified from the plasmid p7C6, were joined by triple-fusion PCR (Fig. 1A). Purified PCR products were used to transform competent $B$. subtilis cells and obtained $\sin R$ knock out strain (KO-SinR). The primers are shown in Additional file 1: Table S1.

The method used for site-directed mutagenesis was similar to that used by [32]. The PCR reaction was conducted using the PrimeSTAR HS DNA polymerase (Takara, Japan) and the pHY-P43/SinR plasmid as the template DNA. The strains and primers are shown in Additional file 1: Table S1. The PCR product was digested by DpnI (Takara, Japan) at $37^{\circ} \mathrm{C}$ for $1 \mathrm{~h}$. The PCR product was transformed into competent cells of $E$. coli DH5 $\alpha$. After the sequence verified, the extracted plasmid was transformed into BS168 KO-SinR cells. E97K, Y101L, W104K, R105S, and SinR ${ }^{\text {quad }}$ (Fig. 1B) were obtained for biofilm formation and MK synthesis.

All strains were cultivated in lysogeny broth (LB) liquid culture or on LB agar plates at $37^{\circ} \mathrm{C}$ for genetic experiments. The fermentation medium consisted of $5 \%(\mathrm{w} / \mathrm{v})$ glycerol, $5 \%(\mathrm{w} / \mathrm{v})$ yeast extract, $5 \%(\mathrm{w} / \mathrm{v})$ soy peptone, $0.38 \%(\mathrm{w} / \mathrm{v}) \mathrm{K}_{2} \mathrm{HPO}_{4}$, and $0.16 \%(\mathrm{w} / \mathrm{v}) \mathrm{KH}_{2} \mathrm{PO}_{4}$. Ampicillin $(100 \mathrm{~g} / \mathrm{mL})$ was added to the medium. All chemicals were purchased from Sangon Biotech Co., Ltd (Shanghai, China). MK-7 standard was purchased from ChromaDex (Irvine, CA, USA). Soy peptone, glycerol, and yeast extract were purchased from Sinopharm Chemical Reagent Co., Ltd (Shanghai, China). Methanol, dichloromethane, 2-propanol, and n-hexane were obtained from Sigma-Aldrich (St. Louis, MO, USA).

\section{Determination of biofilm biomass and MK-7 yield}

Overnight cultures were diluted 1:100 into fermentation medium in a 24-well microtiter plate. After incubation at $37^{\circ} \mathrm{C}$ for $24 \mathrm{~h}$ under microaerobic conditions, the planktonic cells were removed, and the biofilms were washed once with distilled water and then stained by the addition of $0.4 \%$ crystal violet $(\mathrm{CV})$ for $30 \mathrm{~min}$ at room temperature. $\mathrm{CV}$ was then removed with a pipette and biofilms were washed twice with distilled water, solubilized in $30 \%$ acetic acid and measured at $\mathrm{OD}_{570 \mathrm{~nm}}$ as described previously [33]. Three replicates were examined, and the data were analyzed by the Student's $t$-test. The MK-7 detection method was the same as in our previous research [34].

\section{Determination of membrane potential}

Membrane potential was determined as described previously [35]. Briefly, BS168 and SinR ${ }^{\text {quad }}$ were incubated at $37{ }^{\circ} \mathrm{C}$ for 6 days. After cultivation, the cultures were cooled on ice for $10 \mathrm{~min}$, and the cells were harvested by centrifugation $\left(10 \mathrm{~min}, 8000 \mathrm{rpm}, 4{ }^{\circ} \mathrm{C}\right)$. Competent cells were prepared and bacterial suspension was centrifuged (10 $\mathrm{min}, 8000 \mathrm{rpm}, 4{ }^{\circ} \mathrm{C}$ ). The cells were resuspended in $1 \mathrm{~mL}$ of ice-cold buffer solution [distilled deionized water (DDW), $0.9 \% \mathrm{NaCl}$ or $1 \mathrm{mM} \mathrm{MgCl}_{2}$ ] and $30 \mathrm{mM}$ $\mathrm{DiOC}_{2}(3)$ was added. After being mixed vigorously by vortex, both mixtures were incubated in the dark for $4 \mathrm{~min}$ at room temperature. Finally, the cells were centrifuged $\left(10 \mathrm{~min}, 8000 \mathrm{~g}, 4^{\circ} \mathrm{C}\right)$ and washed twice with icecold DDW and analyzed by flow cytometry.

\section{Detection of NADH and NAD ${ }^{+}$[36]}

$\mathrm{NADH} / \mathrm{NAD}^{+}$levels of $B$. subtilis were measured using a commercially available kit (MAK037, Sigma-Aldrich, USA) according to the manufacturer's instructions. $\mathrm{NAD}^{+}$total $\left(\mathrm{NAD}^{+}\right.$and NADH) or NADH levels were quantified by a colorimetric assay at $450 \mathrm{~nm}$ using a SpectraMax i3x (Molecular Devices, USA). The NADH/ $\mathrm{NAD}^{+}$ratio was calculated using Eq. (1):

$$
\text { ratio }=\mathrm{C}_{\mathrm{NADH}} /\left(\mathrm{C}_{\text {total }}-\mathrm{C}_{\mathrm{NADH}}\right)
$$

\section{RNA isolation, library construction, and sequencing}

The fermentation broth of BS168 and SinR ${ }^{\text {quad }}$ was collected on day 6 . The samples were immediately centrifuged for $10 \mathrm{~min}$ at $5000 \mathrm{rpm}$, and then stored at $-80^{\circ} \mathrm{C}$. RNA was extracted by the RNAlock reagent. Total RNA was collected by TRIzol reagent (Invitrogen, USA) and then treated with RNase-free DNase set (NEB, Ipswich, MA, CA, USA). The extracted RNA was detected with an Agilent Bioanalyzer 2100 (Agilent Technologies, Palo Alto, CA, USA). After adding Ribo-Zero Reaction Buffer and Ribo-Zero rRNA Removal Solution (Grampositive bacteria), the volume was fixed to $40 \mu \mathrm{L}$ and the reaction was carried out at $68{ }^{\circ} \mathrm{C}$ for $10 \mathrm{~min}$. The sample was then placed at room temperature for $5 \mathrm{~min}$. The processed RNA was added to the pre-washed magnetic beads, mixed thoroughly, incubated at room temperature for $5 \mathrm{~min}$ and then $50{ }^{\circ} \mathrm{C}$ for $5 \mathrm{~min}$, and then immediately placed on a magnetic stand for more than $1 \mathrm{~min}$. The supernatant was removed, and $180 \mu \mathrm{L}$ water was added to the pellet. Then, $3 \mathrm{M}$ sodium acetate, glycogen $(10 \mathrm{mg} / \mathrm{mL})$, and absolute ethanol $(600 \mu \mathrm{L})$ were added to 


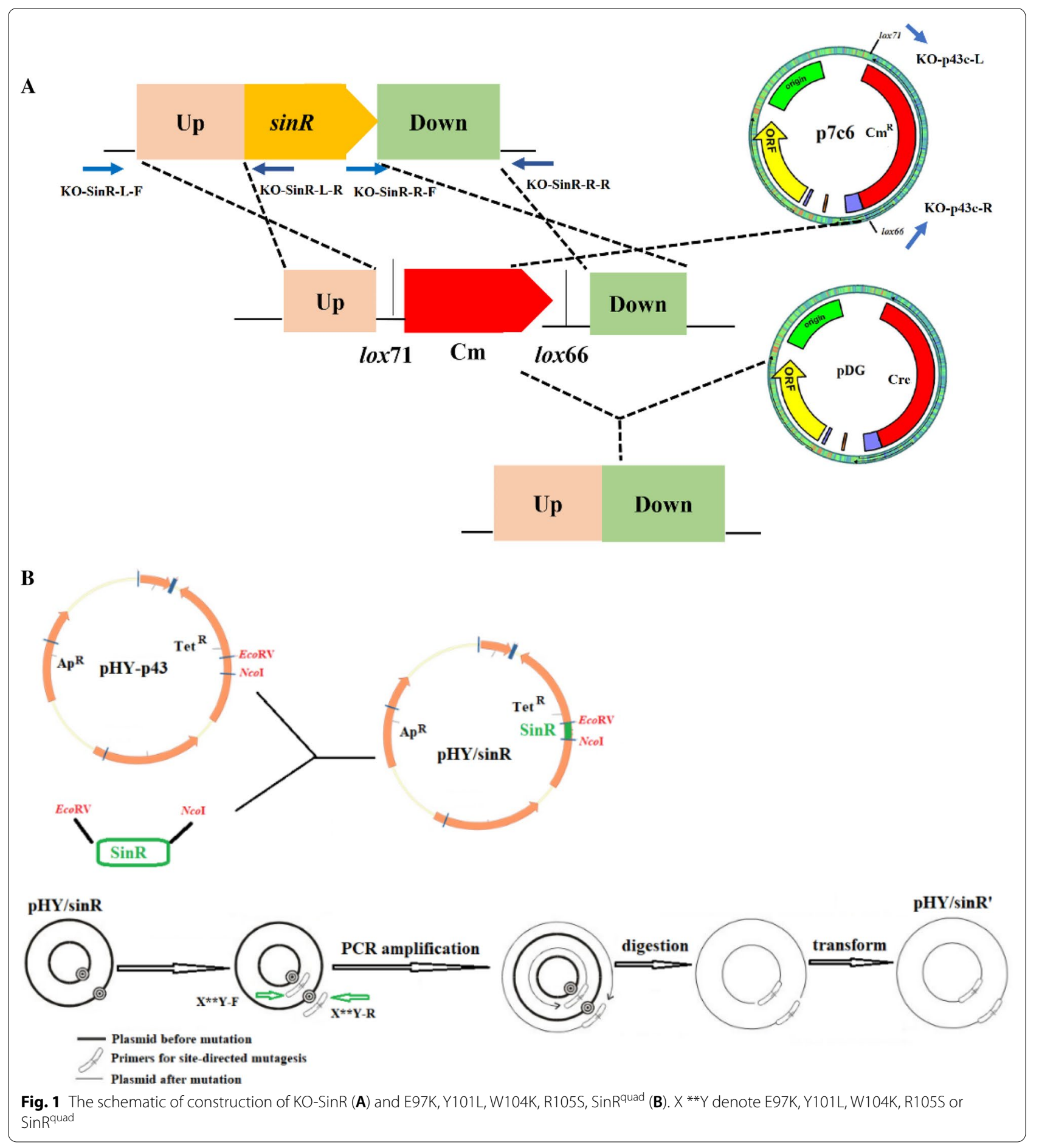

the solution, which was then placed at $-20^{\circ} \mathrm{C}$ for more than $1 \mathrm{~h}$. The solution was centrifuged to obtain a precipitate, which was then dissolved in water to form rRNAdepleted RNA. The RNA library construction from samples at $6 \mathrm{~h}$ was completed by the Shanghai Human Genome Research Center. A $2 \times 150$ bp paired-end sequencing was performed using HiSeq 2000 platform (Illumina, CA, USA) [37].

\section{RNA-seq data analyses}

Clean data was obtained by filtering the original data through SeqPrep/Sickle software to remove the adapter 
reads, and low-quality and short fragments. Then, clean data were mapped to reference sequences through the Bowtie software based on the Burrows-Wheeler method [38]. The RPKM method was used to estimate the expression levels of gene and transcript by RSEM software [39]. Differentially expressed genes (DEGs) between BS168 and SinR ${ }^{\text {quad }}$ were identified by a mathematical statistical model using the edgeR software [40]. The edgeR software can automatically identify and reduce the impact of library construction and sequencing to control the false discovery rate (FDR) [41]. The DEGs were judged through the $\log 2$ ratio $\geq 1$ and FDR of $\leq 0.05$. Then the DEGs were mapped to terms in the gene ontology (GO) and KEGG databases for analyzing the functions and pathways. This analysis of GO functional enrichment used Goatools software with the Fisher precise test method [42]. GO terms with a corrected $p$-value less than 0.05 were regarded as GO terms with significant enrichment in DEGs. In addition, KOBAS software was used for the enrichment analysis of the KEGG pathways [43]. The KEGG pathways with a corrected $p$-value $\leq 0.05$ were significantly enriched in DEGs.

\section{Results}

Site-directed mutagenesis of the transcriptional regulator $\sin R$ decreases biofilm formation and increases MK-7 synthesis in BS168

Previous studies found that biofilm formation was beneficial for synthesizing MK-7 in B. subtilis [4, 44]. Therefore, we were interested in examining which genes play an important role in affecting both biofilm formation and MK synthesis. A comprehensive mutant library of $B$. subtilis was generated, and the biofilm formation ability and MK-7 synthesis of all mutants were tested (Fig. 2). Our initial screening indicated that deletion of the $\sin R$ gene resulted in an increased biofilm phenotype in which the biofilm biomass increased 2.8-fold compared to the wild-type (Fig. 2A, B). Furthermore, the concentration of MK-7 increased 2.6-fold after 6 days of cultivation (Fig. 2C). Therefore, the $\sin R$ gene was chosen for further study.

Some researchers have found that biofilm formation is beneficial for MK-7 synthesis [44], while others said that biofilm formation increased the viscosity, reduced the mass transfer, and subsequently decreased the production of MK-7 [45]. To explore whether there could be a better way to improve MK-7 production by affecting

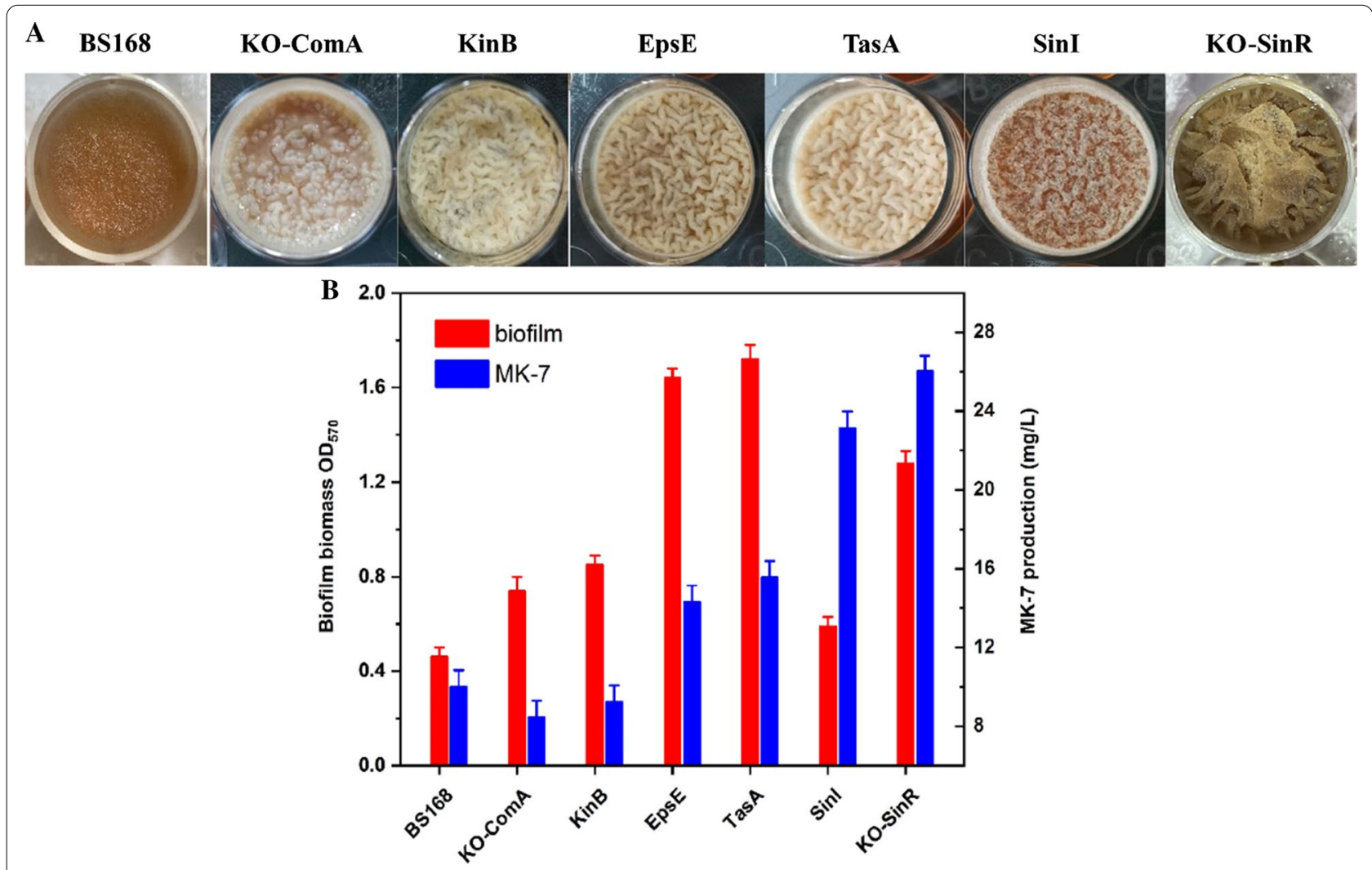

Fig. 2 The morphological changes of the biofilm (A), the biofilm biomass (B) and MK-7 production (C) of comA, $\sin R$ knock out strains, and kinB, epsE, tas $A, \sin /$ overexpression strains 
biofilm formation, we used site-directed mutation to alter the ability of SinR to regulate the extracellular matrix.

Amino acid residues Glu97, Tyr101, Trp104, and Arg105 in SinR (Fig. 3A) were replaced with Lys34, Leu38, Lys41, and Ser42 in SinI (Fig. 3B) to examine the effects on B. subtilis. Figure 4 shows the different biofilm morphologies, biofilm biomasses, and MK-7 production of seven different strains (BS168, E97K, Y101L, W104K, R105S, SinR quad, and KO-SinR) after 6 days of incubation. The B. subtilis 168 wild-type strain (BS168) made the biofilm look smooth with a few wrinkles. The $\mathrm{OD}_{570 \mathrm{~nm}}$ value and the MK-7 production were $0.46 \pm 0.04$ and
$10 \pm 0.84 \mathrm{mg} / \mathrm{L}$, respectively. In contrast, a visibly rough and dry biofilm was found in the KO-SinR strain. The $\mathrm{OD}_{570 \mathrm{~nm}}$ value and the MK-7 production were $1.28 \pm 0.05$ and $26 \pm 0.78 \mathrm{mg} / \mathrm{L}$, respectively. In addition, E97K, Y101L, W104K, and R105S formed more wrinkles than BS168 but fewer wrinkles than KO-SinR. The $\mathrm{OD}_{570 \mathrm{~nm}}$ value of E97K, Y101L, W104K, and R105S increased slightly compared to BS168 but was lower than KO-SinR. However, MK-7 production in four mutants, especially E97K, increased obviously $(61.02 \pm 0.84 \mathrm{mg} / \mathrm{L})$, which was 2.35-fold that of KO-SinR. When the four sites were mutated simultaneously, we obtained the SinR ${ }^{\text {quad }}$ strain.

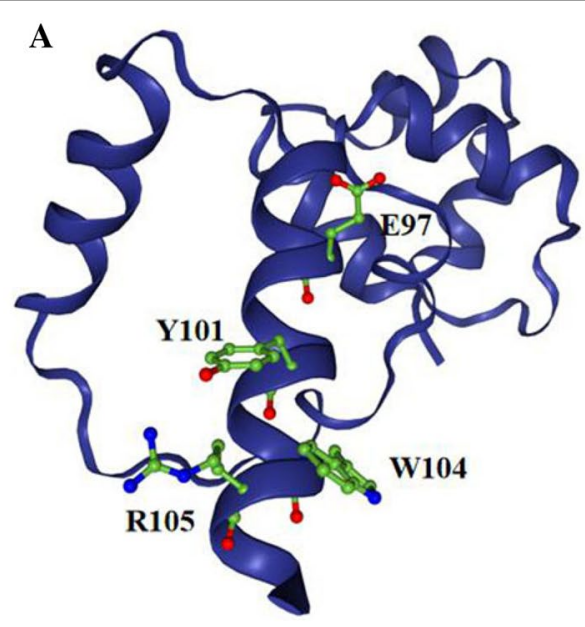

B

SinR 65 KHETEYDGQLDSEWEKLVRDAMTSGVSKKQFREFLDYQKWRKS 107

SinI 2 KNAKQEHFELDQEWVELMVEAKEANISPEEIRKYLLLNKKSAH 44

C
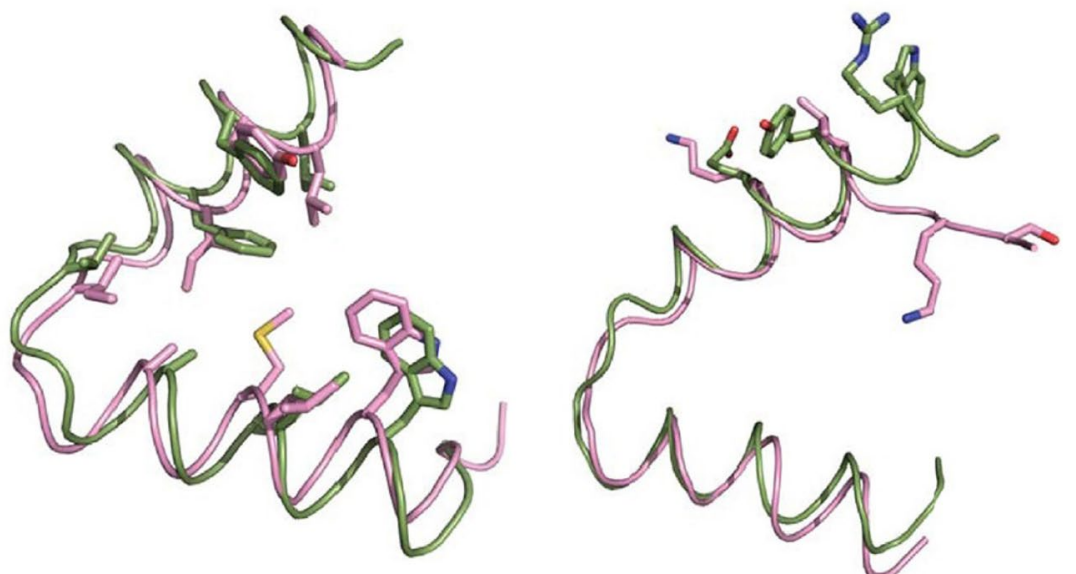

Fig. 3 Structure of SinR and the residues of E97, Y101, W104 and R105 (A), sequence alignment of the helical hook regions of SinR and Sinl (B), Structural alignment of SinR (green) and $\operatorname{Sinl}$ (pink) helical hooks (C). Residues are colored based on sequence similarity from Clustal Omega. Stars denote the residues involved in formation of the SinR tetramer interface. These residues in SinR were mutated to the corresponding residue of Sin to generate the $\operatorname{SinR}^{\text {quad }}$ mutant. Highly conserved amino acids are shown in grey shadow depending on similarity. $\mathbf{C}$ Left, conserved residues line the dimerization interface. Right, SinR residues necessary for tetramer formation are significantly different in Sinl 


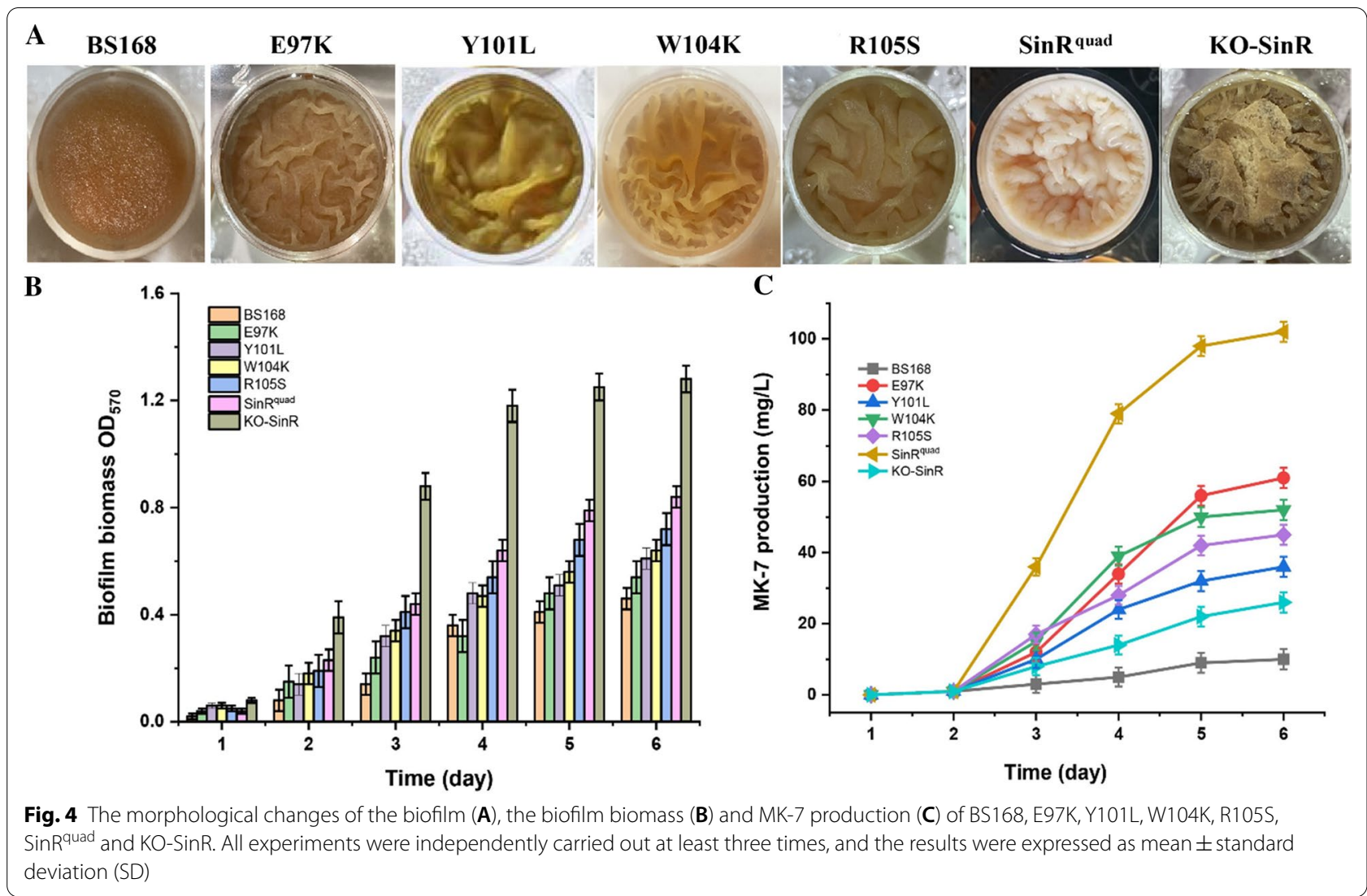

Compared to KO-SinR, SinR quad formed a more wrinkly but smoother biofilm. Although the highest biofilm biomass was obtained by KO-SinR, the maximum MK-7 value $(102.56 \pm 2.84 \mathrm{mg} / \mathrm{L})$ was obtained by $\operatorname{Sin} R^{\text {quad }}$, which was ten times that of BS168, indicating that the increase in MK-7 production was not induced by biomass growth.

\section{Illumina HiSeq mRNA sequencing and functional classification of unigenes}

In order to explore how SinR ${ }^{\text {quad }}$ affects biofilm formation and MK-7 production in B. subtilis, the Illumina RNAseq method was used to link genes in related metabolic pathways affected by site-directed mutagenesis of the transcriptional regulator $\sin R$. The results showed significant changes in expression levels in 1875 of the 3740 identical genes (Fig. 5B, C). Of these, 958 genes were upregulated, and 917 genes were downregulated (Fig. 5C, D). The functional differences were mainly in "membrane", "carbohydrate metabolic process", and "sporulation" (Fig. 6A). The 20 different enrichment pathways included ABC transporters, flagellar assembly, bacterial chemotaxis, ubiquinone and other terpenoid-quinone biosynthesis, and oxidative phosphorylation (Fig. 6B). Because the pathway of the $\mathrm{ABC}$ transporters and phosphotransferase system are associated with the state of the cell membrane [46], these differences indicate changes in the external morphology of the colony, menaquinone biosynthesis, respiratory system, and state of the cell membrane in response to site-directed mutagenesis of $\sin R$.

\section{Differential gene expression related to the QS system and biofilm}

To explore why site-directed mutagenesis of $\sin R$ caused changes in the external morphology of the biofilm, the expression of the QS system and biofilm-related genes of the BS168 and SinR ${ }^{\text {quad }}$ were studied. B. subtilis uses ComX and CSP QS system to regulate the competence and sporulation processes. It can be seen from Fig. 7B, with regard to the CSP QS system of SinR ${ }^{\text {quad }}$, that the expression levels of $\operatorname{spoOA}, a b r B, \sin I$, and $\sin R$ were unchanged. The expression of $\operatorname{slr} R$ was upregulated 1.93-fold. The expressions of tapA, tasA, and epsE were upregulated 9.79-, 0.95-, 4.42-fold, respectively. The upregulation of $\operatorname{tap} A, \operatorname{tas} A$, and epsE implied that the extracellular matrix, which constituted the biofilm, was promoted in SinR ${ }^{\text {quad }}$.

With regard to the ComX QS system, the expressions of $\operatorname{com} X, \operatorname{com} A$, and $\operatorname{com} S$ were unchanged, indicating that expression of the ComX QS system and ComX 

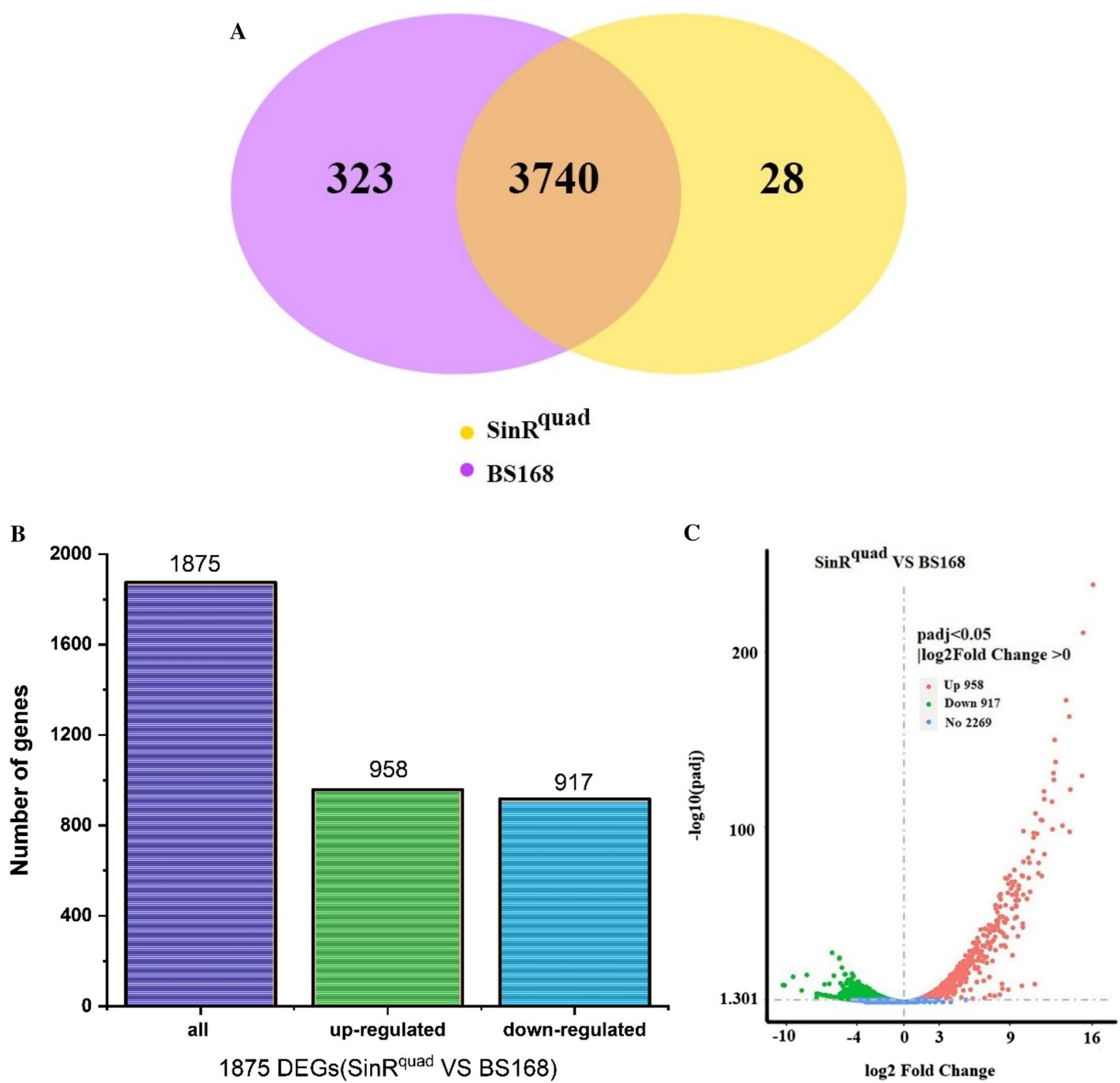

Fig. 5 Summary of draft reads of samples by Illumina deep sequencing. A Global comparison of BS168 and SinR ${ }^{\text {quad }}$ samples by Venn diagrams. B Number of up-regulated and down-regulated DEGs of the BS168 and SinR ${ }^{\text {quad }}$ samples. C Visualization of differential genes (Volcano-plots)

regulated the biofilm genes and were unaffected by sitedirected mutagenesis of SinR.

Numerous previous studies have reported that SinR is a positive effector of motility and cell separation, which is not conducive to the formation of biofilms [47]. However, it was still unknown whether the expression of flagella and chemotaxis proteins would change with sitedirected mutagenesis of SinR. Therefore, the expression levels of the $s w r A$ and hag genes were investigated. As we know, swrA encodes proteins for the hook-basal body of flagella, chemotaxis, and the flagellum-associated sigma factor $\sigma^{\mathrm{D}}$ [48]. The hag gene, a late-flagellar gene, encodes the flagellar filament structural protein flagellin, which is responsible for swimming motility [49]. Figure 7B shows that the relative transcription of the $s w r A$ gene had no change between SinR ${ }^{\text {quad }}$ and BS168. However, expression of the hag gene was downregulated by $75.65 \%$. Therefore, site-directed mutagenesis of E97K, Y101L, W104K, and R105S of $\operatorname{sinR}$ hindered the expression of the late-flagellar gene. 


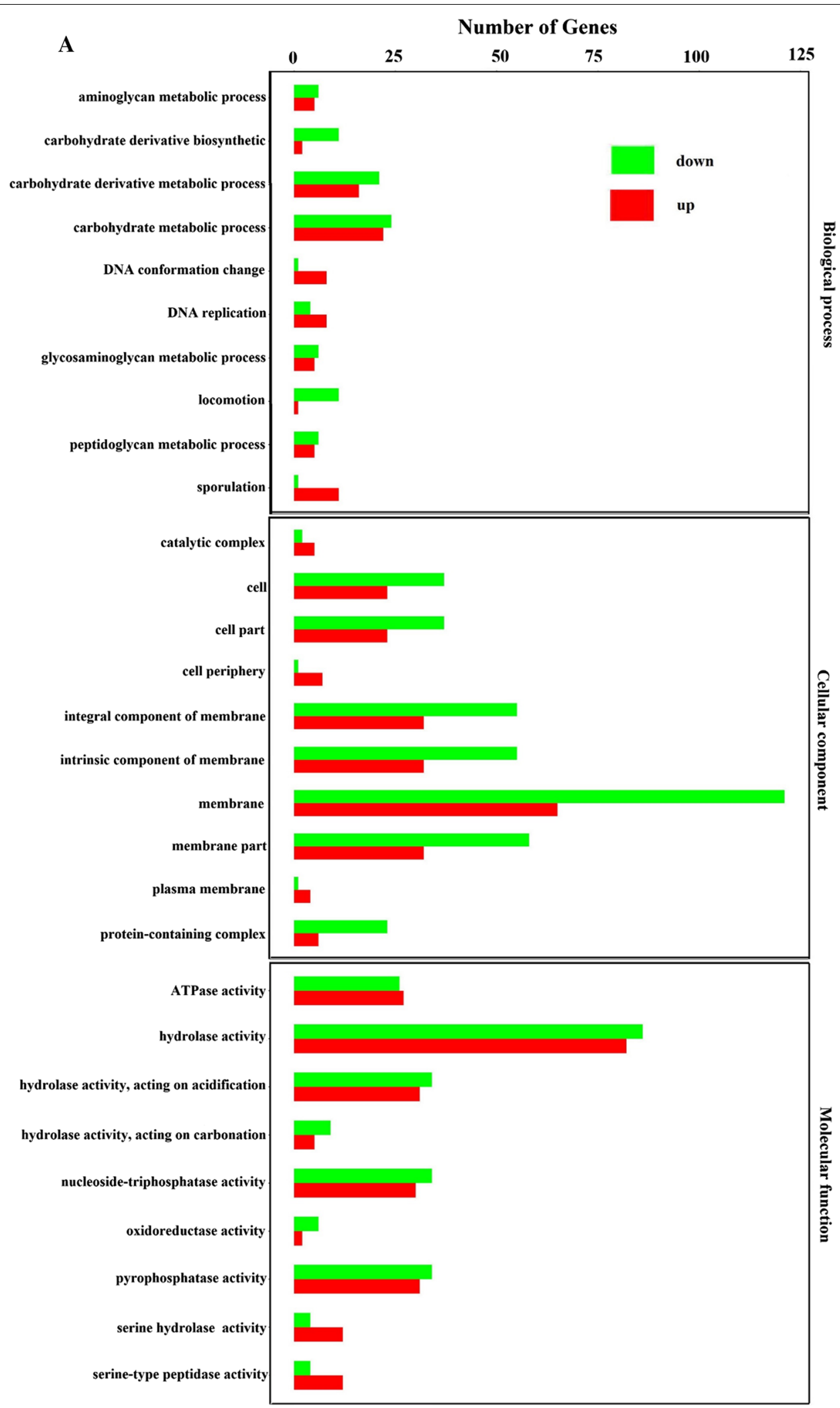

Fig. 6 Transcriptomics analysis of differences between BS168 and SinR ${ }^{\text {quad }}$. A Gene Ontology (GO) functional analysis of differential genes. Unique sequences were assigned to three categories: molecular functional, cellular components and biological process. B Kyoto Encyclopedia of Genes and Genomes (KEGG) pathway analysis of functions of differential genes. All experiments were independently carried out at least three times, and the results were expressed as mean \pm standard deviation (SD) 


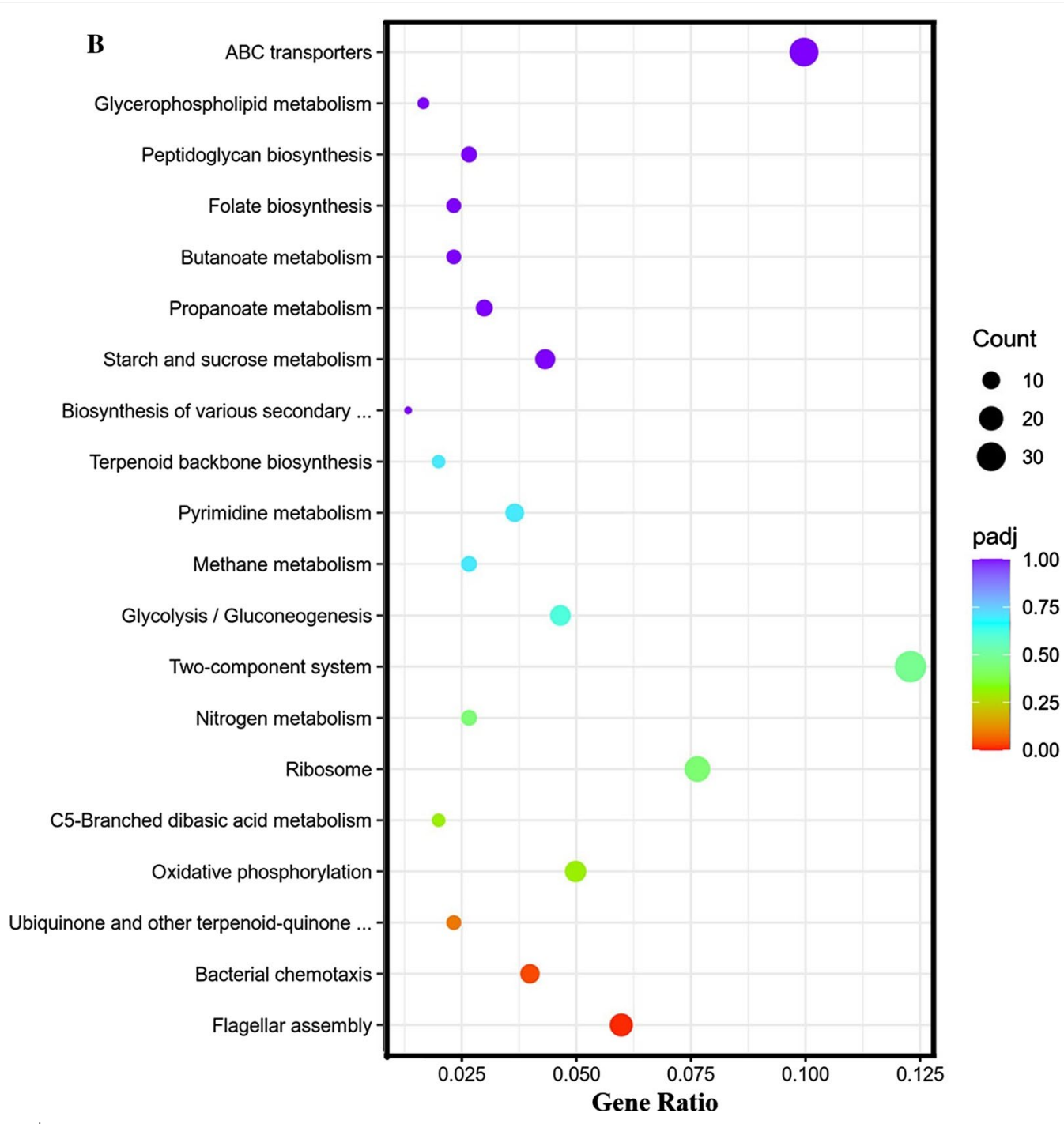

Fig. 6 continued

The appD gene, which encodes an oligopeptide $\mathrm{ABC}$ transporter (ATP-binding protein) [50], was upregulated 4.07-fold, implying that membrane transport was promoted in Sin $R^{\text {quad }}$. Therefore, SinR ${ }^{\text {quad }}$ promoted the synthesis of extracellular polymeric substances through the CSP QS system, improved membrane transport, inhibited the swimming motility of late-flagellar, and, thus, promoted the biofilm biomass.

\section{Differential gene expression related to MK-7 biosynthesis}

The biosynthesis pathway of MK-7 in B. subtilis presented in Fig. 8 can be categorized into four modules, namely the glycerol metabolism pathway (Module I), the methylerythritol phosphate (MEP) pathway
(Module II), the shikimate (SA) pathway (Module III), and the MK-7 pathway (Module IV) [51].

Previous studies comparing the effect of four carbon sources (i.e., soluble starch, sucrose, glucose, and glycerol) on MK-7 synthesis and growth of B. subtilis natto found that the presence of glycerol in the media resulted in higher MK-7 production [10, 52]. Therefore, the first step in the biosynthesis pathway was the uptake of glycerol, which is catalyzed in an energy-independent manner by a membrane channel protein, the glycerol facilitator (GlpF) [53]. The main pathway of glycerol dissimilation involves a glycerol kinase (GlpK) that phosphorylates glycerol to glycerol-3-phosphate (Gly$3 \mathrm{P})$, and a Gly-3P dehydrogenase (GlpD) that oxidizes Gly-3P to dihydroxyacetone phosphate (DHAP), an 

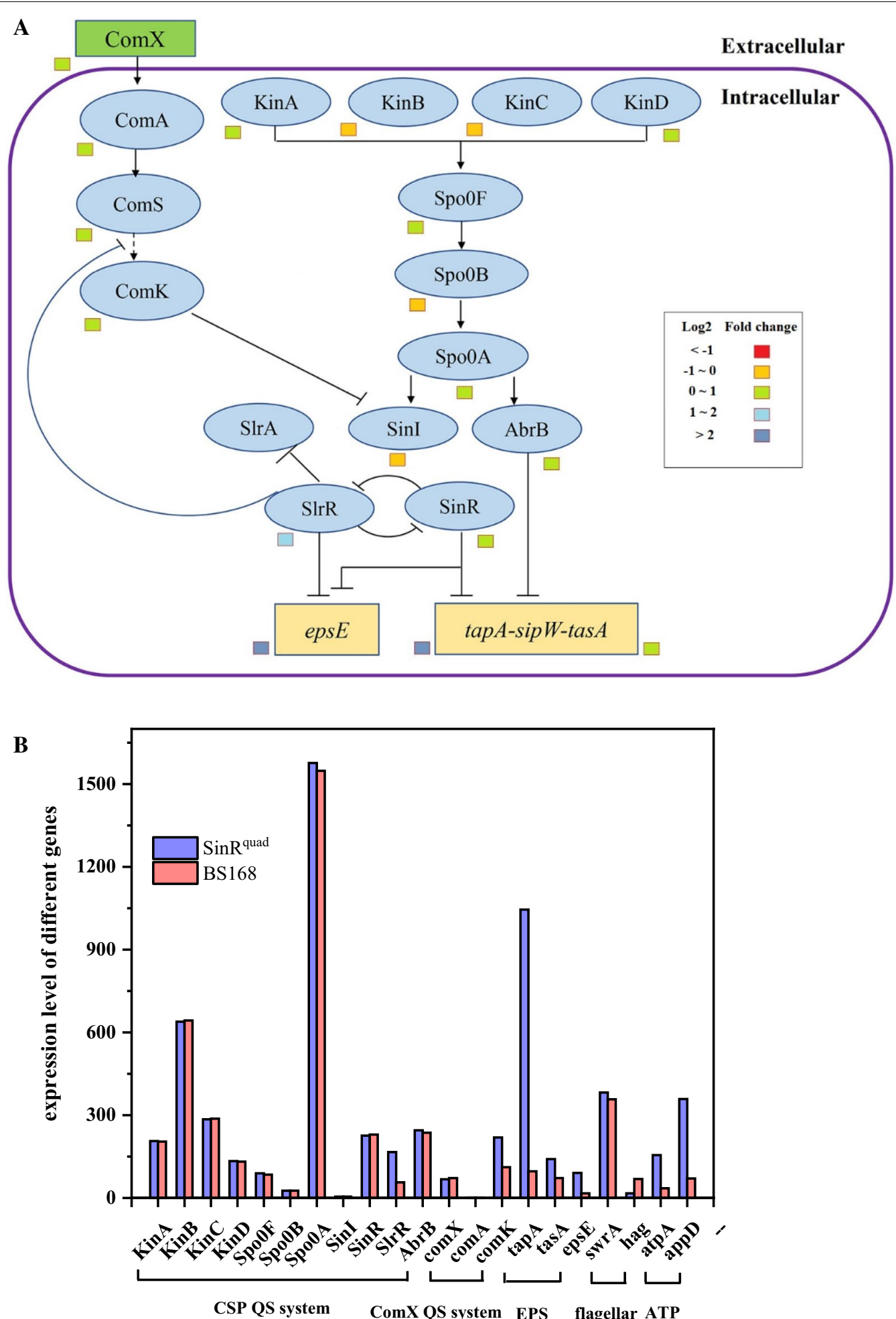

Fig. 7 Changes in transcript abundance of genes involved in quorum sensing (QS) system and biofilm between BS168 and SinR Ruad samples. A Transcriptional regulation of the biofilm. B The expression level of biofilm forming related genes of SinR ${ }^{\text {quad }}$ compared with BS168 


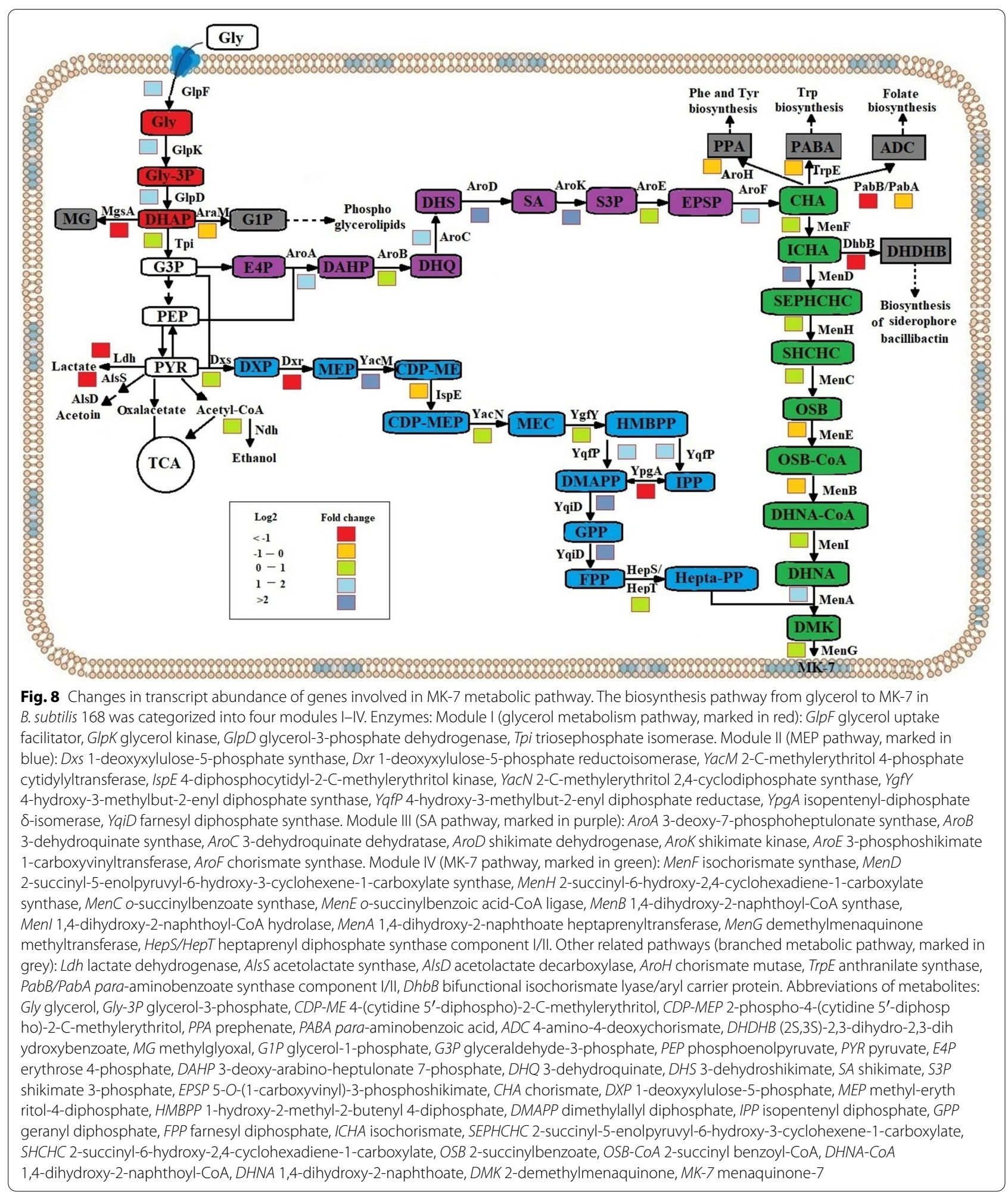

intermediate in glycolysis. In the glycerol metabolism pathway, the expression of $g l p F, g l p k$, and $g l p D$ were upregulated 1.21-, 2.20-, and 1.11-fold, respectively. In addition, methylglyoxal synthase (MgsA) and glycerol1-phosphate dehydrogenase (AraM), which catalyze the conversion of DHAP to methylglyoxal (MG) and 
the reduction of DHAP to glycerol-1-phosphate (G1P), were downregulated by $97 \%$ and $46 \%$, respectively. The result indicated that $\operatorname{SinR}^{\text {quad }}$ could increase the consumption of the substrate glycerol and weaken the other two branch pathways of DHAP, allowing DHAP to flow into glycolysis as much as possible.

Module II provides isopentenyl diphosphate (IPP) and its isomer dimethylallyl diphosphate (DMAPP) for the biosynthesis of isoprenoids, which is the precursor of the side chain of MK-7. However, studies on the key enzymes in the MEP pathway in B. subtilis are rare. Figure 8 shows that most of the enzymes in Module II were upregulated in the SinR ${ }^{\text {quad }}$ strain. As the first rate-limiting enzyme in the MEP pathway, 1-deoxy-D-xylulose 5-phosphate reductoisomerase (Dxs), which catalyzes the reaction of pyruvate and glyceraldehyde 3-phosphate to form 1-deoxy-D-xylose-5-phosphate (DXP), was upregulated 0.91 -fold. The result was consistent with a previous study that found overexpressing $d x s$ could increase the yield of isoprene by $40 \%$ over that of the wild-type B. subtilis [54]. In addition, Yqfp, which catalyzes the synthesis of isopentenyl diphosphate (IPP) and its isomer dimethylallyl diphosphate (DMAPP), was upregulated 1.95-fold. Especially, YacM and Yqid, which catalyze the reaction of MEP and cytosine triphosphate (CTP) to form 4-diphosphocytidyl-2-C-methyl-D-erythritol (CDP-ME), and polymerize IPP to farnesyl pyrophosphate (FPP), were upregulated 2.26-fold and 2.06-fold, respectively.

Module III provided chorismate (CHA), the precursor of the main chain of MK-7. CHA is essential in cellular metabolism for providing the precursors for the biosynthesis of three aromatic amino acids: tyrosine (Tyr), phenylalanine (Phe), and tryptophan (Trp) [55]. The four enzymes AroA, B, C, and D can catalyze D-erythrose 4-phosphate and phosphoenolpyruvate to form shikimate. Shikimate will be converted to chorismate by AroK, E, and F. Figure 7 shows that aro $A, B, C, D, K, E$, and $F$ were upregulated 1.47-, 0.75-, 1.64-, 3.77-, 8.30-, 1.08-, and 0.14-fold, respectively. The genes encoding AroH, TrpE, PabA, and PabB, which are involved in the synthesis of three aromatic amino acids, were downregulated by $41 \%, 29 \%, 21 \%$, and $62 \%$, respectively. The result indicated that AroA, D, and $\mathrm{K}$ played an important role in CHA synthesis. This was consistent with a previous study showing that simultaneous overexpression of aro $A$ and $\operatorname{aroK}$ in $B$. subtilis resulted in a twofold increase in MK-7 compared with strain BS168 [56].

Module IV is the last pathway and is for MK-7 synthesis. Seven enzymes (MenF, D, H, C, E, B, I) for the synthesis of 1,4-dihydroxy-2-naphthoyl-CoA (DHNA-CoA) in the MK-7 pathway were detected with different expression changes (Additional file 2: Table S2). Five genes were upregulated, especially men $D$ whose expression level was highly regulated by 3.87 -fold. Finally, both $\operatorname{men} A$ and men $G$ - which could combine the isoprene side chain and naphthoquinone ring and then catalyze the methylation to form MK-7-were upregulated 2.03- and 0.72-fold, respectively, in the SinR ${ }^{\text {quad }}$ strain. It is consistent with our previous studies where overexpression of menG/ubiE in Elizabethkingia meningoseptica enhanced the MK content 1.41-fold [34]. These results provide a new idea for further understanding the effect of $\operatorname{SinR}^{\text {quad }}$ on the biosynthesis of MK-7 at the transcriptional level.

\section{Differential gene expression related to the cell membrane}

MK-7 is a component of bacterial cell membranes and plays an important role in electron transfer and respiration. A schematic of electron flow mediated by MK in B. subtilis is illustrated in Fig. 9A. Respiration occurs in the cell membrane of Gram-positive bacteria. Electron donors, with the help of an enzyme, transfer two electrons to MK and cytochrome c. MK and cytochrome c, with the help of another enzyme, in turn transfer these two electrons to an electron acceptor oxygen to form water. To determine changes in electron transfer and respiration in the cell membrane of dead cells (Control), BS168, and SinR quad, the membrane potential, the expression of genes encoding the cytochromes, and MK-7 production were measured after 6 days of cultivation. We observed that the mean fluorescence intensity (MFI) of $\operatorname{SinR}^{\text {quad }}$ rose to 86 , which was 1.8 -fold that of BS168 (Fig. 9B, C). A rise in the magnitude of the membrane potential is referred to as electrical hyperpolarization in SinR ${ }^{\text {quad. }}$. In addition, the expression levels of the $c t a C-G$ operator and $q c r A-C$ operator, which encode the cytochromes [57], were upregulated (Fig. 9D), and the Log2 fold changes were all greater than 0 . MK-7 production was also ten times that of BS168 (Fig. 4C). These results proved that $S_{i n R^{\text {quard }}}$ could deliver more electrons and promote respiration in B. subtilis. A previous study found that electrons were formed during the conversion of oxalate to formate and $\mathrm{CO}_{2}$ [58]. Therefore, we tested the expression level of oxalate-decarboxylase $(\mathrm{OxdC})$, which, with the help of $\mathrm{Mn}$ and $\mathrm{O}_{2}$, catalyzes the conversion of oxalate to formate and $\mathrm{CO}_{2}$. However, the expression level of $o x d C$ demonstrated a $45 \%$ decrease. In addition, the expression levels of $f d h D$ and $y r h E$, which encode formate dehydratase and are used to oxidize formate to $\mathrm{CO}_{2}$ and electrons, were downregulated by $60 \%$ and $94 \%$, indicating that electrical hyperpolarization of SinR ${ }^{\text {quard }}$ was not due to this process. Therefore, we speculate that there may be other processes that can donate large amounts of electrons. It has been reported that $\mathrm{NADH}$ is the most important electron donor. It donates electrons under the action of NADH dehydrogenase and transfers electrons to the electron transport system 


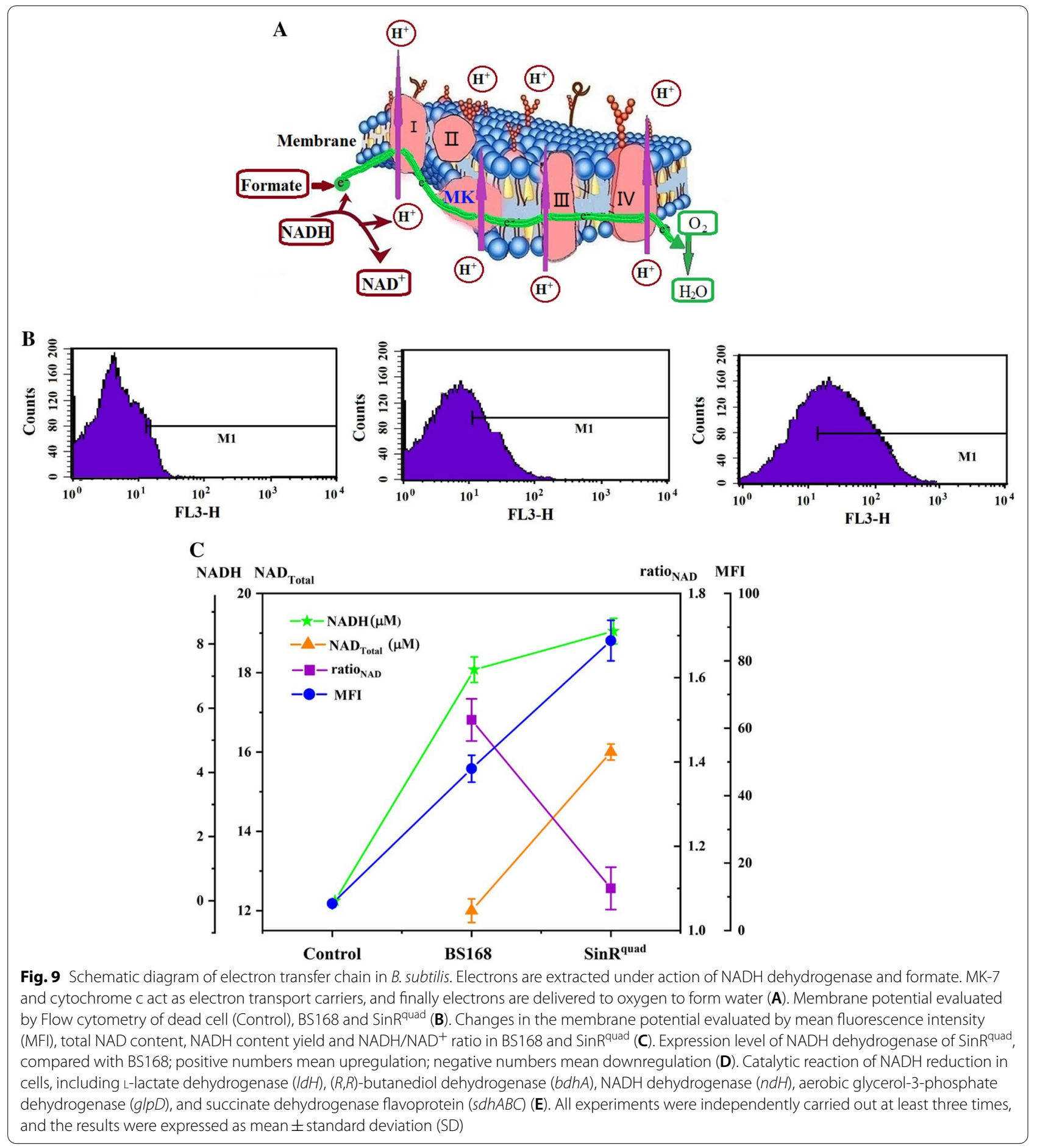

(ETM) and pumps protons out of the cell [36]. The process of producing electrons is shown in Fig. 9E. Consequently, $\mathrm{NAD}^{+}$total $\left(\mathrm{NAD}^{+}\right.$and $\left.\mathrm{NADH}\right)$ and $\mathrm{NADH}$ levels and the $\mathrm{NADH} / \mathrm{NAD}^{+}$ratio were quantified by a colorimetric assay. The $\mathrm{NAD}^{+}$total and NADH levels increased 1.33- and 1.17-fold, respectively, while the
$\mathrm{NADH} / \mathrm{NAD}^{+}$ratio decreased by $27 \%$. This result was consistent with a previous study, which found that the ratio of NADH/NAD ${ }^{+}$in B. subtilis was inversely proportional to the concentration of MK-7 [36]. The expression levels of NADH dehydrogenases (i.e., $n d H, b d h A, s d h A$ $C$, $i d H$, and $g l p D$ ) were also tested. As shown in Fig. 9D, 
D

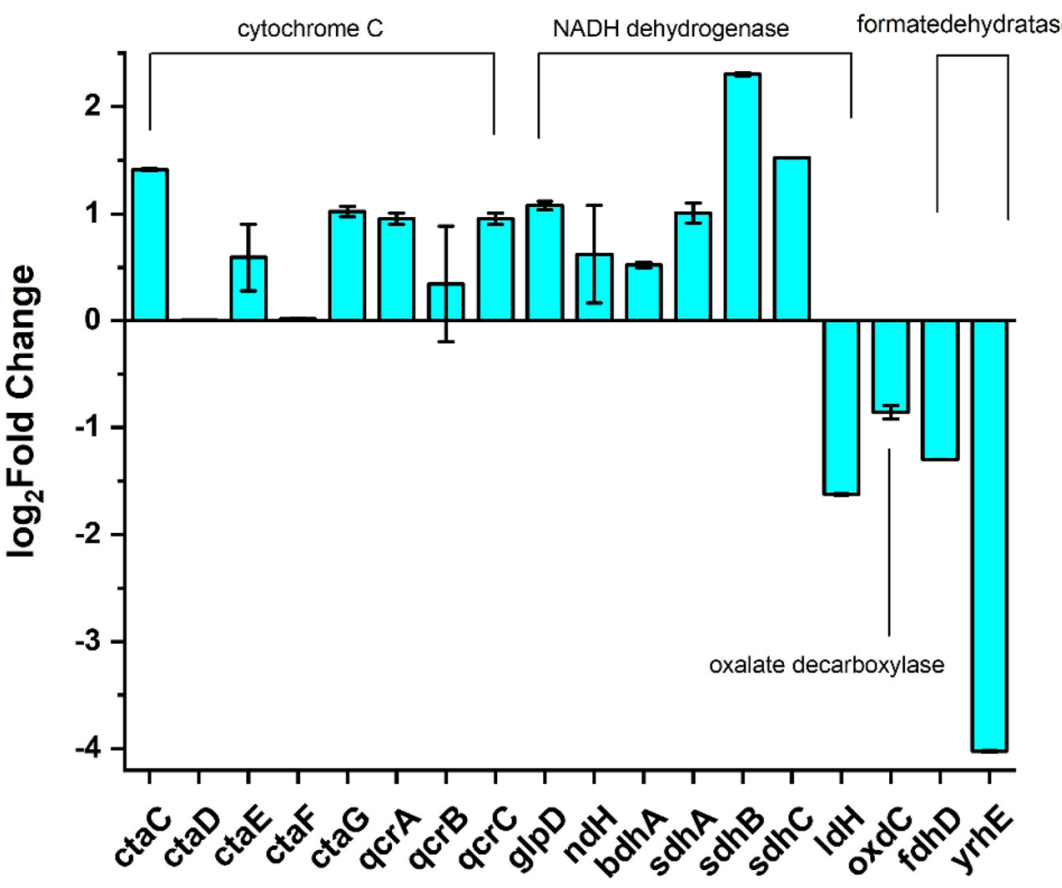

E
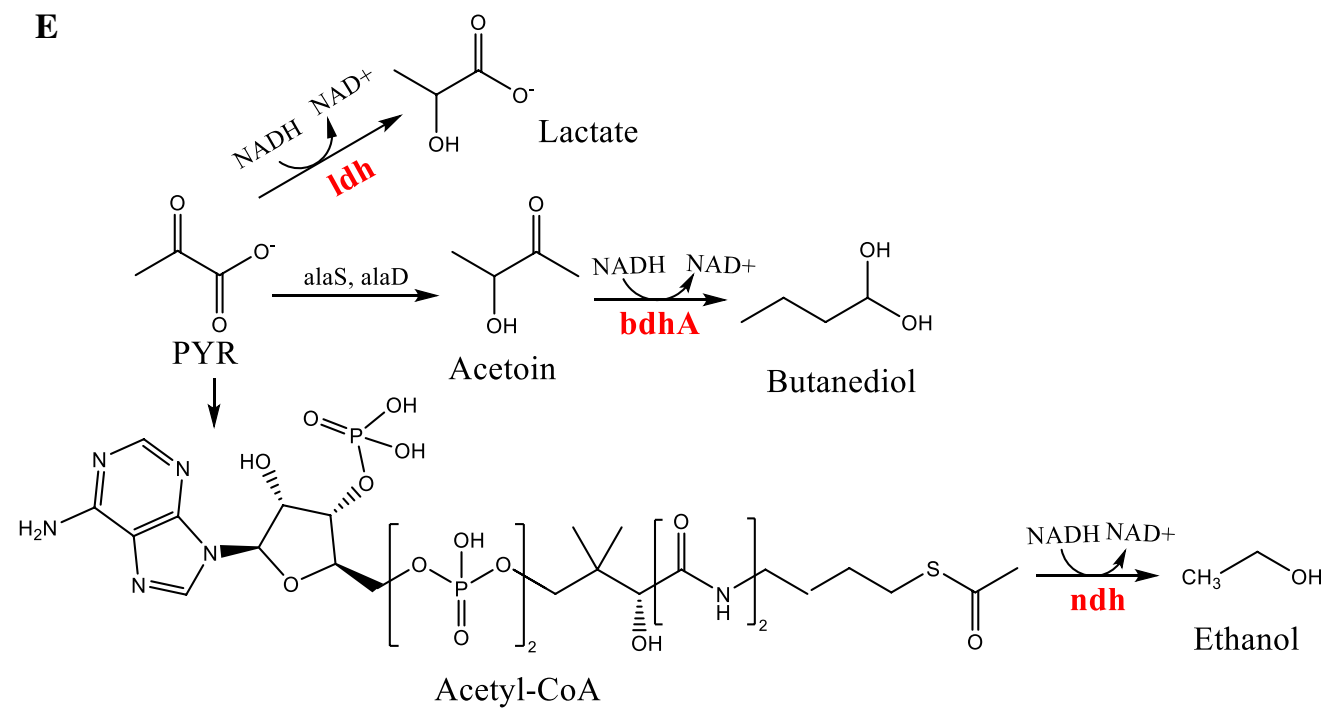

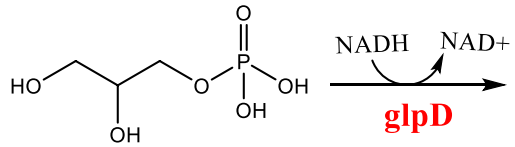

Gly-3P

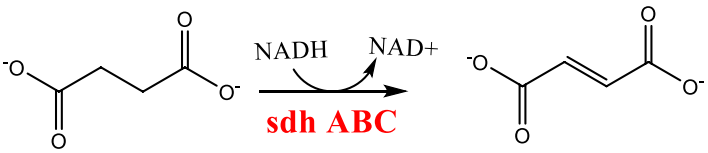

Succinate<smiles>O=C(CO)COP(=O)(O)O</smiles>

DHAP

Fumarate

Fig. 9 continued 
the expression levels of most NADH dehydrogenases were upregulated. Especially, $s d h A, s d h B, s d h C$, and $g l p D$, increased 1.01-, 3.93-, 1.87-, and 1.11-fold, respectively.

\section{Discussion}

Previous studies have found that biofilm formation is beneficial for MK-7 synthesis in B. subtilis [44]. However, others supposed that biofilm formation increases the viscosity, reduces the mass transfer, and subsequently decreases MK-7 production [45]. To explore whether MK-7 synthesis is affected by biofilm formation and whether there is a quantitative correlation between MK-7 synthesis and biofilm formation, we used transcriptomic analysis to target SinR (a QS system transcriptional regulator). We revealed many potential genes related to MK-7 biosynthesis, spore and biofilm formation, and the respiratory oxidation system in B. subtilis. As far as we know, this study is the first report to illustrate the relationship between active sites of SinR and MK-7 biosynthesis, biofilm formation, and the respiratory oxidation system in B. subtilis. This work also provides new information for the further enhancement of MK-7 production, which is essential for the industrialized application of MK-7.

As we know, $\operatorname{SinR}$ is a constitutively expressed transcriptional regulator and has been identified as a master regulator of biofilm formation [46]. DNA binding by SinR leads to repression of the epsA-O and tapA-sipW-tasA operons by the CSP QS system [46], both of which are involved in the production of biofilm matrix polysaccharides and proteins. Our study confirmed this conclusion since KO-SinR caused an obviously increased biofilm phenotype, and the biofilm biomass increased 2.8-fold compared to the wild-type (Fig. 2A, B).

SinI is an antagonist of SinR by forming SinI-SinR deadend complex and sequestering free SinR, thereby ensuring biofilm formation. A previous study found that SinI displaces a SinR monomer from its homotetramer complex [59]. Despite the SinR dimer interface and the SinI dimer interface being highly homologous in sequence and structure (Fig. 3B, C), the four key residues involved in SinR tetramer formation (E97, Y101, W104, R105) are significantly different in SinI. Furthermore, Glu97 and Arg105 as well as pi-stacking from Tyr101 and Trp104 were found to form salt bridges to join adjacent helices [60]. Therefore, Glu97, Tyr101, Trp104, and Arg105 in SinR were replaced with Lys34, Leu38, Lys41, and Ser42 in SinI and mimicked the SinR tetramer interface with DNA binding. A previous study found that equal molar ratios of SinR and SinI resulted in a complete disruption in the SinR homotetramer with a broad $1.7 \mathrm{~S}$ distribution [60]. Our results verified the prediction that when SinR is replaced by a SinR mutation, the SinR homotetramer may be disrupted, which affects SinR DNA-binding activity and promotes biofilm formation. Five mutants (E97K, Y101L, W104K, R105S, and SinR ${ }^{\text {quad }}$ ), especially SinR ${ }^{\text {quad }}$, which contain four point mutations and thus disrupt the SinR homotetramer more severely, formed more wrinkly and smoother biofilms compared to BS168.

Different biofilm morphologies formed by site-directed mutagenesis of SinR affect the bacteria to suit outside environmental changes and nutrient transport. SinR ${ }^{\text {quad }}$ formed a more wrinkled biofilm surface compared to BS168 and the other five mutants, while the wrinkles formed a network of interconnected channels with a low resistance to liquid flow. Pressure within the channels was less than the atmospheric pressure outside. This pressure gradient could drive flow through the channels, facilitate nutrient flow through the biofilm [61], and finally affect the synthesis of secondary metabolites. In our study, we found the expressions of $g l p F, g l p k$, and $g l p D$ in SinR ${ }^{\text {quad }}$ were upregulated 1.21-, 2.20-, and 1.11fold, respectively, indicating that $\operatorname{Sin}^{\text {quad }}$ formed a more winkled biofilm that facilitated glycerol flow through the biofilm. A previous study found that the glycerol dissociation pathway is important for MK synthesis. When $g l p K$ and $g l p D$ were overexpressed, MK-7 titer had a $10 \%$ increase [51]. KO-SinR could also form a wrinkled biofilm (Fig. 3A). However, the biofilm surface was drier than the other mutants, which means that motile cells become spores that were not beneficial for metabolite synthesis. This was one reason why MK-7 synthesis by SinR ${ }^{\text {quad }}$ and E97K were 10-times and 2.35-times higher than KO-SinR.

Transcriptomic analysis showed that the pathways such as $\mathrm{ABC}$ transporters, bacterial chemotaxis, and oxidative phosphorylation changed significantly, indicating that the respiratory system and state of the cell membrane were changed obviously in SinR ${ }^{\text {quad }}$. A previous study discovered that changing the state of the membrane in Escherichia coli could increase the synthesis of MK-8 [62]. Our study verified this prediction by quantitatively determining the membrane potential and respiration of SinR ${ }^{\text {quad }}$ and BS168. MFI of SinR ${ }^{\text {quad }}$ rose to 86, which was 1.8-fold that of BS168 (Fig. 9B, C). A rise in the magnitude of the membrane potential is referred to as electrical hyperpolarization in Sin ${ }^{\text {quad, }}$, which means more protons and electrons were produced in SinR $\mathrm{R}^{\text {quad }}$ compared to BS168. The extra electrons were donated by NADH under the action of NADH dehydrogenase and transferred to the electron transport system (ETM). This was indicated by the 1.33- and 1.17-fold increase in $\mathrm{NAD}^{+}$total and NADH levels, respectively. The expression levels of most NADH dehydrogenases were upregulated. In particular, $s d h A, s d h B, s d h C$, and $g l p D$, increased 1.01-, 3.93-, 1.87-, 1.11-fold, respectively. The increase in 
electron and electrical hyperpolarization stimulated the synthesis of the electron transport chain components, such as cytochrome $\mathrm{c}$ and $\mathrm{MK}$, to ensure electron transfer efficiency [44]. Active ETM was another reason why MK-7 synthesis in SinR ${ }^{\text {quad }}$ was ten times higher than in KO-SinR and BS168.

Many researchers have found that MenA and MenD play an important role in $\mathrm{MK}$ synthesis in different strains $[51,63]$. Our study also found that the expression of $m e n A$ and $m e n D$ increased 2.04 times and 3.87 times in $\operatorname{Sin} R^{\text {quad }}$, respectively, confirming again that the step connecting the isoprene side chain and naphthoquinone ring by MenA, and the catalysis of isochorismate (ICHA) to 2-succinyl-5-enolpyruvyl-6-hydroxy-3-cyclohexene1-carboxylate (SEPHCHC) by MenD, were the rate-limiting steps. More glyceraldehyde 3-phosphate entering the MEP pathway is beneficial for the formation of the polyprenyl side chain, and entering the SA pathway is conducive to the synthesis of the naphthoquinone ring. According to previous research, the biosynthesis of the polyprenyl side chain, rather than biosynthesis of the naphthoquinone ring (1,4-dihydroxy-2-naphtoic acid), limited total MK production in Lactococcus lactis [64]. However, in our study, we found most of the genes in the biosynthesis of the naphthoquinone ring were upregulated and more glyceraldehyde 3-phosphate entered the SA pathway. A possible reason could be that different strains have different metabolic regulation patterns in the MK-7 metabolic pathway. Therefore, the regulatory mechanisms of this pathway need to be further explored.

In summary, we preliminarily determined the mechanism of how site-directed mutagenesis of the transcriptional regulator SinR affects the biosynthesis of menaquinone in Bacillus subtilis. We did this by analyzing the QS system, biofilm formation, cell membrane state, and MK-7 synthesis pathway of SinR ${ }^{\text {quad }}$ and BS168. E97K, Y101L, W104K, R105S, and SinR ${ }^{\text {quad }}$ disrupted the SinR homotetramer, thus forming a more wrinkly and smoother biofilm than BS168. On the one hand, the more wrinkled and smoother biofilm facilitated glycerol flow through the biofilm and enhanced MK-7 synthesis. On the other hand, electrical hyperpolarization stimulated the synthesis of the electron transport chain components, such as cytochrome $\mathrm{c}$ and MK, to ensure electron transfer efficiency.

\section{Supplementary Information}

The online version contains supplementary material available at https://doi. org/10.1186/s12934-021-01603-5.

Additional file 1: Table S1. Strains and primers used in this work

Additional file 2: Table S2. Genes significantly changed in the SinRquad compared to Bacillus subtilis 168

\section{Acknowledgements}

We want to thank Prof. Long Liu and Dr. Shixiu Cui (Jiangnan University, China) for their generous donation of the plasmid P7Z6 and strain B. subtilis 168 .

\section{Authors' contributions}

JW: conceptualization, writing—original draft, investigation, visualization. WL: methodology, writing - review and editing. S-gz: methodology, resources. S-hQ: validation, supervision. ZW: methodology, writing—review and editing. M-jZ: methodology, writing - review and editing. Methodology, W-sH: writing - review and editing. JW: methodology, writing —-review and editing. L-xH: validation, supervision. YL: supervision, project administration. Z-IX: supervision, writing - review and editing. All authors read and approved the final manuscript.

\section{Funding}

The study was supported by the National Nature Science Foundation of China (No. 31871781, 31772081), Anhui university natural science research key project (No. KJ2018A0106), Science and technology project of Wuhu (2020yf05), the National Undergraduate Innovation and Entrepreneurship Program (No. 2016103630055, 201810363046) and Anhui Provincial Undergraduate Innovation and Entrepreneurship Program (No. 201710363178).

\section{Availability of data and materials}

The data generated and analyzed during this study are included in the article and its Additional files 1 and 2 .

\section{Declarations}

Ethics approval and consent to participate

This manuscript does not involve the data collected from animals or humans.

\section{Consent for publication}

All authors consent to publish this manuscript.

\section{Competing interests}

The authors declare that there is no conflict of interests.

\section{Author details}

${ }^{1}$ College of Biology and Food Engineering, Anhui Polytechnic University, Wuhu 241000, China. ${ }^{2}$ Anhui Engineering Laboratory for Industrial Microbiology Molecular Breeding, Wuhu 241000, China. ${ }^{3}$ Wuhu Zhanghengchun Medicine CO., LTD, Wuhu 241000, China.

Received: 8 April 2021 Accepted: 31 May 2021

Published online: 07 June 2021

\section{References}

1. Berenjian A, Mahanama R, Kavanagh J, Dehghani F. Vitamin K series: current status and future prospects. Crit Rev Biotechnol. 2015;35(2):199-208.

2. Zhao C, Wan Y, Tang G, Jin Q, Zhang H, Xu Z. Comparison of different fermentation processes for the vitamin $\mathrm{K}_{2}$ (Menaquinone-7) production by a novel Bacillus velezensis ND strain. Process Biochem. 2021;102:33-41.

3. Hu X, Liu M, Luo M, Ren L, Ji X, Huang H. Enhancing menaquinone-7 production by Bacillus natto R127 through the nutritional factors and surfactant. Appl Biochem Biotechnol. 2017;182(4):1630-41.

4. Berenjian A, Chan NLC, Mahanama R, Talbot A, Regtop H, Kavanagh J, Dehghani F. Effect of biofilm formation by Bacillus subtilis natto on Menaquinone-7 biosynthesis. Mol Biotechnol. 2013;54(2):371-8.

5. Fujimoto N, Kosaka T, Yamada M. Menaquinone as well as ubiquinone as a crucial component in the Escherichia coli respiratory chain. Chem Biol. 2012;17:187-208

6. Sato T, Schurgers $\sqcup$, Uenishi K. Comparison of menaquinone-4 and menaquinone-7 bioavailability in healthy women. BioMed Central. 2012;11(1).

7. Iwamoto J. Vitamin $\mathrm{K}_{2}$ therapy for postmenopausal osteoporosis. Nutrients. 2014;6(5):1971-80.

8. Gröber U, Reichrath J, Holick MF, Kisters K. Vitamin K: an old vitamin in a new perspective. Dermatoendocrinology. 2014;6(1):e968490. 
9. Ravishankar B, Dound YA, Mehta DS, Ashok BK, de Souza A, Pan MH, Ho CT, Badmaev A, Vaidya ADB. Safety assessment of menaquinone-7 for use in human nutrition. J Food Drug Anal. 2015;23:99-108.

10. Berenjian A, Mahanama R, Talbot A, Biffin A, Regtop H, Valtchev P, Kavanagh J, Dehghani F. Efficient media for high menaquinone-7 production: response surface methodology approach. New Biotechnol. 2011;28(6):665-72.

11. Mahanama R, Berenjian A, Valtchev $P$, Talbot A, Biffin R, Regtop $H$, Dehghani F, Kavanagh JM. Enhanced production of menaquinone 7 via solid substrate fermentation from Bacillus subtilis. Int J Food Eng. $2011 ; 7(5): 1-23$

12. Wu W, Ahn BY. Improved menaquinone (Vitamin $\mathrm{K}_{2}$ ) production in cheonggukjang by optimization of the fermentation conditions. Food Sci Biotechnol. 2011;20(6):1585-91.

13. Sato T, Yamada Y, Ohtani Y, Mitsui N, Murasawa H, Araki S. Efficient production of menaquinone (vitamin $\mathrm{K}_{2}$ ) by a menadione-resistant mutant of Bacillus subtilis. J Ind Microbiol Biotechnol. 2001;26:115-20.

14. Sun X, Liu H, Wang P, Wang L, Ni W, Yang Q, Wang H, Tang H, Zhao G, Zheng Z. Construction of a novel MK-4 biosynthetic pathway in Pichia pastoris through heterologous expression of HsUBIAD1. Microb Cell Fact. 2019;18:1-16.

15. Yuan P, Cui S, Liu Y, Li J, Lv X, Liu L, Du G. Combinatorial engineering for improved menaquinone-4 biosynthesis in Bacillus subtilis. Enzyme Microb Tech. 2020;141:1-9.

16. Liu Y, Liu L, Li J, Du G, Chen J. Synthetic biology toolbox and chassis development in Bacillus subtilis. Trends Biotechnol. 2019;37:548-62.

17. Mahdinia E, Demirci A, Berenjian A. Biofilm reactors as a promising method for vitamin K (menaquinone-7) production. Appl Microbiol Biot. 2019;103:5583-92.

18. Dong X, Li N, Liu Z, Lv X, Shen Y, Li J, Du G, Wang M, Liu L. CRISPRi-guided multiplexed fine-tuning of metabolic flux for enhanced lacto- $N$-neotetraose production in Bacillus subtilis. J Agric Food Chem. 2020;68:2477-84.

19. Anesiadis N, Cluett WR, Mahadevan R. Dynamic metabolic engineering for increasing bioprocess productivity. Metab Eng. 2008;10:255-66.

20. Xu P. Production of chemicals using dynamic control of metabolic fluxes. Curr Opin Biotechnol. 2018;53:12-9.

21. Tan S, Prather K. Dynamic pathway regulation: recent advances and methods of construction. Curr Opin Chem Biol. 2017:41:28-35.

22. Xu P, Li L, Zhang F, Stephanopoulos G, Koffas M. Improving fatty acids production by engineering dynamic pathway regulation and metabolic control. Proc Natl Acad Sci. 2014;111:11299-304.

23. Zhang F, Carothers J, Keasling J. Design of a dynamic sensor-regulator system for production of chemicals and fuels derived from fatty acids. Nat Biotechnol. 2012;30:354-9.

24. Zhang F, Keasling J. Biosensors and their applications in microbial metabolic engineering. Trends Microbiol. 2011;19:323-9.

25. Dahl R, Zhang F, Alonso-Gutierrez J, Baidoo E, Batth T, Redding-Johanson A, Petzold C, Mukhopadhyay A, Lee T, Adams P, Keasling J. Engineering dynamic pathway regulation using stress-response promoters. Nat Biotechnol. 2013;31:1039-46.

26. Wan X, Marsafari M, Xu P. Engineering metabolite-responsive transcriptional factors to sense small molecules in eukaryotes: current state and perspectives. Micro Cell Fact. 2019;18:1-12.

27. Lv Y, Qian S, Du G, Chen J, Zhou J, Xu P. Coupling feedback genetic circuits with growth phenotype for dynamic population control and intelligent bioproduction. Metab Eng. 2019;54:109-16.

28. Lyon G, Novick R. Peptide signaling in Staphylococcus aureus and other Gram-positive bacteria. Peptides. 2004;25:1389-403.

29. Shen Y, Fong L, Yan Z, Liu J. Combining directed evolution of pathway enzymes and dynamic pathway regulation using a quorum-sensing circuit to improve the production of 4-hydroxyphenylacetic acid in Escherichia coli. Biotechnol Biofuels. 2019;12:1-11.

30. Miller MB, Bassler BL. Quorum sensing in bacteria. Annu Rev Microbiol. 2001;55:165-99.

31. Yan X, Yu H, Hong Q, Li S. Cre/lox system and PCR-based genome engineering in Bacillus subtilis. Appl environ microb. 2008;74:5556-62.

32. Liu Y, Ding X, Xue Z, Hu L, Cheng Q, Chen M, Su Y, Zhu B, Xu P. Sitedirected mutagenesis of UbiA to promote menaquinone biosynthesis in Elizabethkingia meningoseptica. Process Biochem. 2017;58:186-92.
33. Zhu B, Ge X, Stone V, Kong X, El-Rami F, Liu Y, Kitten T, Xu P. ciaR impacts biofilm formation by regulating an arginine biosynthesis pathway in Streptococcus sanguinis SK36. Sci Rep. 2017;7:17183.

34. Liu Y, Yang Z, Xue Z, Qian S, Wang Z, Hu L, Zhou S, Wang J, Zhu H, Ding X, Yu F. Influence of site-directed mutagenesis of UbiA, overexpression of $d x r$, menA and $u b i E$, and supplementation with precursors on menaquinone production in Elizabethkingia meningoseptica. Process Biochem. 2018;68:64-72

35. Novo DJ, Perlmutter NG, Hunt RH, Shapiro HM. Multiparameter flow cytometric analysis of antibiotic effects on membrane potential, membrane permeability, and bacterial counts of Staphylococcus aureus and Micrococcus luteus. Antimicrob Agents Chemother. 2000;44(4):827-34.

36. Wang $H$, Liu H, Wang L, Zhao G, Tang H, Sun X, Ni W, Yang Q, Wang $P$, Zheng $Z$. Improvement of menaquinone-7 production by Bacillus subtilis natto in a novel residue-free medium by increasing the redox potential. Appl Microbiol Biotechnol. 2019;103:7519-35.

37. Cheng P, Zhu S, Lu J, Hu X, Ren L. Transcriptomic analysis of gene expression of menaquinone-7 in Bacillus subtilis natto toward different oxygen supply. Food Res Int. 2020;137:109700.

38. Langmead B, Salzberg SL. Fast gapped-read alignment with Bowtie2. Nat Methods. 2012;9(4):357-9.

39. Li B, Dewey CN. RSEM: accurate transcript quantification from RNASeq data with or without a reference genome. BMC Bioinformatics. 2011;12(1).

40. Trapnell C, Hendrickson DG, Sauvageau M, Goff L, Rinn JL, Pachter L. Differential analysis of gene regulation at transcript resolution with RNA-seq Nat Biotechnol. 2013:31:46-53.

41. Reiner A, Yekutieli D, Benjamini Y. Identifying differentially expressed genes using false discovery rate controlling procedures. Bioinformatics. 2003;19:368-75.

42. Benjamini Y, Hochberg Y. Controlling the false discovery rate: a practical and powerful approach to multiple testing. J R Stat Soc Series B (Methodological). 1995;57(1):289-300.

43. Xie C, Mao X, Huang J, Ding Y, Wu J, Dong S. KOBAS 2.0: a web server for annotation and identification of enriched pathways and diseases. Nucleic Acids Res. 2011:39:W316-22.

44. Cui S, Xia H, Chen T, Gu Y, Lv X, Liu Y, Li J, Du G, Liu L. Cell membrane and electron transfer engineering for improved synthesis of menaquinone-7 in Bacillus subtilis. iScience. 2020;23(3):100918.

45. Berenjian A, Mahanama R, Talbot A, Regtop H, Kavanagh J, Dehghani F. Designing of an intensification process for biosynthesis and recovery of menaquinone-7. Applied Biochem Biotech. 2014;172:1347-57.

46. Zhou A, Cao Y, Zhou D, Hu S, Tan W, Xiao X, Li X. Global transcriptomic analysis of Cronobacter sakazakii CICC 21544 by RNA-seq under inorganic acid and organic acid stresses. Food Res Int. 2020;130:108963.

47. Joseph NA, Cecilia R, Richard LJ. Molecular basis of the activity of SinR protein, the master regulator of biofilm formation in Bacillus subtilis. J Biol Chem. 2013;288:10766-78.

48. Aizawa S, Zhulin IB, MarqIn-Magana L, Ordal GW, Sonenshein AL, Hoch JA, Losick R. In Bacillus subtilis and its closest relatives: chemotaxis and motility. Washington, DC: American Society for Microbiology Press; 2001. p. 437-52.

49. Mirel DB, Chamberlin MJ. The Bacillus subtilis flagellin Gene (hag) is transcribed by the sigma-28 form of RNA-polymerase. J Bacteriol. 1989;171:3095-101.

50. Kunst F, Ogasawara N, Moszer I, Albertini AM, Alloni M, Azevedo V, Bertero MG, Bessieres P, Bolotin A, Borchert S, Borriss R, Boursier L, Brans A, Braun M, Brignell SC, Bron S, Brouillet S, Bruschi CV, Caldwell B, Capuano V, Carter NM, Choi SK, Cordani JJ, Connerton IF, Cummings NJ, Danie RA, Denziot F, Devine KM, Dusterhoft A, Ehrlich SD, Emmerson PT, Entian KD, Errington J, Fabret C, Ferrari E, Foulger D, Fritz C, Fujita M, Fujita Y, Fuma S, Galizzi A, Galleron N, Ghim SY, Glaser P, Goffeau A, Golightly EJ, Grandi G, Guiseppi G, Guy BJ, Haga K, Haiech J, Harwood CR, Henaut A, Hilbert H, Holsappel S, Hosono S, Hullo MF, Itaya M, Jones L, Joris B, Karamata D, Kasahara Y, Klaerr-Blanchard M, Klein C, Kobayashi Y, Koetter P, Koning stein G, Krogh S, Kumano M, Kurita K, Lapidus A, Lardinois S, Lauber J, Lazarevic V, Lee SM, Levine A, Liu H, Masuda S, Mauel C, Medigue C, Medina N, Mellado RP, Mizuno M, Moestl D, Nakai S, Noback M, Noone D, Reilly MO, Ogawa K, Ogiwara A, Oudega B, Park SH, Parro V, Pohl TM, Portelle D, Porwollik S, Prescott AM, Presecan E, Pujic P, Purnelle B, Rapoport G, Rey M, Reynolds S, Rieger M, Rivolta C, Rocha E, Roche B, Rose M, 
Sadaie Y, Sato T, Scanlan E, Schleich S, Schroeter R, Scoffone F, Sekiguch J, Sekowska A, Seror SJ, Serror P, Shin BS, Soldo B, Sorokin A, Tacconi E, Takagi T, Takahashi H, Takemaru K, Takeuchi M, Tamakoshi A, Tanaka T, Terpstra P, Togoni A, Tosato V, Uchiyama S, Andebol MV, Vannier F, Vassarotti A, Viari A, Wambutt R, Wedler H, Weitzenegger T, Winters P, Wipat A, Yamamoto H, Yamane K, Yasumoto K, Yata K, Yoshida K, Yoshikawa HF, Zumstein E, Yoshikawa H, Danchin A. The complete genome sequence of the gram-positive bacterium Bacillus subtilis. Nature. 1997;390:249-56.

51. Yang S, Cao Y, Sun L, Li C, Lin X, Cai Z, Zhang G, Song H. Modular pathway engineering of Bacillus subtilis to promote de novo biosynthesis of menaquinone-7. ACS Synth Biol. 2019;8:70-81.

52. Luo M, Ren L, Chen S, Ji X, Huang H. Effect of media components and morphology of Bacillus natto on menaquinone-7 synthesis in submerged fermentation. Biotechnol Bioprocess Eng. 2016;21 (6):777-86.

53. Yeh Jl, Kettering R, Saxl R, Bourand A, Darbon E, Joly N, Briozzo P, Deutscher J. Structural characterizations of glycerol kinase: unraveling phosphorylation-induced long-range activation. Biochemistry. 2009:48:346-56.

54. Xue J, Ahring BK. Enhancing isoprene production by genetic modification of the 1-deoxy-D-xylulose-5-phosphate pathway in Bacillus subtilis. Appl Environ Microbiol. 2011;77:2399-405.

55. Krämer M, Bongaerts J, Bovenberg R, Kremer S, Müller U, Orf S, Wubbolts $M$, Raeven L. Metabolic engineering for microbial production of shikimic acid. Metab Eng. 2003;5:277-83.

56. Cui S, Lv X, Wu Y, Li J, Du G, Rodrigo LA, Liu L. Engineering a bifunctional Phr60-Rap60-Spo0A quorum-sensing molecular switch for dynamic finetuning of menaquinone-7 synthesis in Bacillus subtilis. ACS Synth Biol. 2019;8:1826-37.

57. Qin Y, He Y, She Q, Larese-Casanova P, Li P, Chai Y. Heterogeneity in respiratory electron transfer and adaptive iron utilization in a bacterial biofilm. Nat Commun. 2019;10(1):3702.
58. Conter C, Oppici E, Dindo M, Rossi L, Magnani M, Cellini B. Biochemical properties and oxalate-degrading activity of oxalate decarboxylase from Bacillus subtilis at neutral pH. IUBMB Life. 2019;71:917-27.

59. Bai U, Mandic-Mulec I, Smith I. Sinl modulates the activity of SinR, a developmental switch protein of Bacillus subtilis, by protein-protein interaction. Genes Dev. 1993;7:139e148.

60. Milton ME, Logan Draughn G, Bobay BG, Stowe SD, Olson AL, Feldmann EA, Thompson RJ, Myers KH, Santoro MT, Kearns DB, Cavanagh J. The solution structures and interaction of SinR and Sinl: elucidating the mechanism of action of the master regulator switch for biofilm formation in Bacillus subtilis. J Mol Biol. 2020;432(2):343-57.

61. Wilking JN, Zaburdaev V, De Volder M, Loscik R, Brenner MP, Weitz DA. Liquid transport facilitated by channels in Bacillus subtilis biofilms. Proc Natl Acad Sci USA. 2013;110(3):848-52

62. Liu Y, Ding X, Xue Z, Hu L, Zhang N, Wang Z, Yang J, Cheng Q, Chen $M$, Zhang $Z$, Zheng $Z$. The change of the state of cell membrane can enhance the synthesis of menaquinone in Escherichia coli. World J Microbiol Biotechnol. 2017;33(3):52.

63. Xu JZ, Yan WL, Zhang WJ. Enhancing menaquinone-7 production in recombinant Bacillus amyloliquefaciens by metabolic pathway engineering. RSC Adv. 2017;7:28527-34.

64. Bøe CA, Holo H. Engineering Lactococcus lactis for increased vitamin $\mathrm{K}_{2}$ production. Front Bioeng Biotech. 2020;8:191.

\section{Publisher's Note}

Springer Nature remains neutral with regard to jurisdictional claims in published maps and institutional affiliations.
Ready to submit your research? Choose BMC and benefit from:

- fast, convenient online submission

- thorough peer review by experienced researchers in your field

- rapid publication on acceptance

- support for research data, including large and complex data types

- gold Open Access which fosters wider collaboration and increased citations

- maximum visibility for your research: over $100 \mathrm{M}$ website views per year

At BMC, research is always in progress.

Learn more biomedcentral.com/submissions 